\title{
Time-resolved pulse propagation in a strongly scattering material
}

\author{
Patrick M. Johnson, ${ }^{1,2, *}$ Arnout Imhof, ${ }^{2}$ Boris P. J. Bret, ${ }^{1,3}$ Jaime Gómez Rivas, ${ }^{1, \dagger}$ and Ad Lagendijk ${ }^{1,3}$ \\ ${ }^{1}$ Van der Waals-Zeeman Instituut, Universiteit van Amsterdam, 1018 XE Amsterdam, The Netherlands \\ ${ }^{2}$ Debye Instituut, Universiteit Utrecht, P.O. Box 80000, 3508 TA Utrecht, The Netherlands \\ ${ }^{3}$ Faculty of Applied Physics and MESA + Research Institute, University of Twente, 7500 AE Enschede, The Netherlands
}

(Received 2 April 2003; published 11 July 2003)

\begin{abstract}
Light transport in macroporous gallium phosphide, perhaps the strongest nonabsorbing scatterer of visible light, is studied using phase-sensitive femtosecond pulse interferometry. Phase statistics are measured at optical wavelengths in both reflection and transmission and compared with theory. The diffusion constant of light is measured in both reflection and transmission as a function of thickness and compared with theories for diffusive transport and localization. An unusually high energy velocity due to the bicontinuous structure of the porous network is reported. For such strongly scattering samples, we show that surface properties and the effective index of refraction need to be treated carefully.
\end{abstract}

DOI: 10.1103/PhysRevE.68.016604

PACS number(s): 42.25.Dd, 42.30.Ms, 61.43.Gt

\section{INTRODUCTION}

The propagation of light in strongly scattering disordered systems continues to excite considerable interest for a number of reasons. Understanding diffusing multiply scattered optical waves has allowed for probing biological tissue through imaging [1], and colloidal dynamics through diffusing wave spectroscopy [2]. Disorder in photonic crystals leads to new and interesting forms of light diffusion [3], while even weak disorder in a photonic band gap can lead to localization [4]. Recently, anisotropic diffusion of light has been measured in both liquid crystals and porous gallium phosphide [5-7]. In the regime of extremely strong scattering, such interference contributions can cause diffusive propagation to break down [8] leading to a number of fascinating effects such as intensity fluctuations [9], universal conductance fluctuations [10], and localization [11]. The regime of localization is difficult to obtain experimentally, thus a host of experimental questions regarding localization remain [12-14].

In disordered systems, light transport can be described by a statistical distribution of random paths through the material. These paths are characterized by a transport mean free path $l$, the distance required to randomize the direction of propagation. In most randomly scattering systems, the interference contribution to the statistical distribution is small. The statistics of such systems can be described by the diffusion equation in which the rate of propagation is characterized by the diffusion constant $D=v_{E} l / 3$, where $v_{E}$ is the energy velocity. There remain a number of open questions regarding diffusive light transport. For example, the effective index of refraction has yet to be explained in strongly scattering samples [15]. Connections between the mesoscopic topology of the sample and propagation properties are also of current interest $[16,17]$. Much of the phase statistics of diffuse waves, while theoretically predicted in detail [18] and

\footnotetext{
*Email address: P.M.Johnson@phys.uu.nl

${ }^{\dagger}$ Present address: Institut Für Halbleitertechnik, RWTH Aachen, Sommerfeldstr. 24, 52074 Aachen, Germany.
}

examined experimentally with microwaves [19], have not been tested at optical wavelengths. Extending such measurements to optical and infrared wavelengths is vital, since at such wavelengths low absorbing samples may be fabricated and possible implications for laser, telecom, and biomedical technologies as well as for random lasers [20] may be explored.

If the scattering strength is increased, interference terms may substantially change the propagation statistics. For example, diffuse light shows nearly twice the intensity when scattered directly backward due to constructive interference of time-reversed paths. This effect, known as enhanced backscattering, offers a way to measure the scattering strength in the sample, $1 /\left(k l_{s}\right)$, where $k=2 \pi n_{e} / \lambda$ is the wave vector in the medium, $n_{e}$ is the effective refractive index of the medium, $\lambda$ is the wavelength, and $l_{s}$ is the scattering mean free path [21]. As one progresses from weak to very strong scattering, there exists a transition from extended states, where $D$ is sample size invariant, to localized states, where $D$ evidences exponential reduction with increasing sample thickness [22]. For three-dimensional systems, this requires strongly scattering materials with $k l_{s} \simeq 1$ [23]. This is a challenging experimental prerequisite, and consequently there are few reports of classical wave localization in threedimensional systems $[12,13]$. The reports have been questioned [14] because residual absorption can masquerade as localization as it too results in an exponential decay of the total transmission with sample thickness. More sensitive probes of the onset of localization may require either direct access to the phase coherence, dynamic measurements, or both [24].

Recently, our group reported the preparation of macroporous networks of gallium phosphide $(\mathrm{GaP})$ fabricated by electrochemical etching $[25,26]$. The single-crystal, bicontinuous mesoscopic topology contrasts with that of commonly studied powders. While powders are a collection of polydisperse, high-index particles, macroporous materials have a spongelike geometry and can achieve low volume to volume ratios of high index to low index material. The samples are strongly scattering in the red, due to the large refractive index of GaP (3.2-3.3), but show an extremely low degree of absorption at this frequency. The samples 
show an inverse relationship between total transmission and sample thickness, consistent with the classical diffusion theory. However, the enhanced backscattering cones show anomalous rounding for the most strongly scattering GaP. Unlike total transmission measurements, which average over all light paths, enhanced backscatter cones probe the path length distribution and may, therefore, be more sensitive to certain statistical features of localization. Thus it was hypothesized that these samples show characteristics of both localization and classical diffusion [27]. Both measurement techniques are static, in that they average the transmitted or backscattered light over time.

In this paper, we report time-resolved, phase-sensitive measurements for these macroporous $\mathrm{GaP}$ samples using a short-pulse interferometric technique [28] in both transmission and reflection. Such measurements allow us to measure $D$ as a function of thickness and thus test for renormalization effects, measure $v_{e}$ for a spongelike geometry, and resolve the dynamic phase statistics at optical wavelengths. We find that even in the presence of enhanced backscatter cone rounding, $D$ did not show the thickness-dependent renormalization characteristic of localization. The samples do, however, show an unusually high value for $v_{e}$ when compared with strongly scattering powders. The phase statistics in transmission confirm predictions of the diffusion theory. In reflection, the predicted functional form for the phase derivative distribution is confirmed while there seems to be a deviation with the predicted thickness-dependent width of this distribution. Also, we show that surface effects for such strongly scattering samples must be carefully treated when measuring bulk properties.

\section{EXPERIMENT}

The samples consisted of a porous layer of $\mathrm{GaP}$ on wafers of single-crystalline GaP. These are the same samples described in Ref. [25], made by anodic etching $n$-type singlecrystalline GaP wafers. The porous region is about $5 \mathrm{~mm}$ in diameter. Etching begins at the surface of the wafer, and proceeds in at a constant rate. Thus the thickness of these samples could be varied from 5 to $120 \mu \mathrm{m}$ by varying the total etching time. This process produces a uniform porosity throughout the thickness of the wafer. After anodic etching, the pores were not of optimum breadth to optimize the scattering cross section. Some optical anisotropy was also present due to the slightly oblong shape of the pores. The anodic etch process also leaves a thin $(\sim 100 \mathrm{~nm})$ solid GaP surface layer where the pores initiated.

To improve the scattering strength, the anodically etched samples were further photoanodically etched. This increased the porosity from $35 \%$ to $50 \%$ and decreased the anisotropy. Scanning electron microscopy images of the resulting structures showed them to be nearly isotropic. The average pore size in these samples was estimated at $132 \pm 30 \mathrm{~nm}$ [25]. Photoanodic etching also reduces the amount of surface material in the samples. This process was sufficient to completely remove the surface layer in the thicker samples. In the thinnest sample, some of the surface layer remained.

The time-resolved measurements were performed using
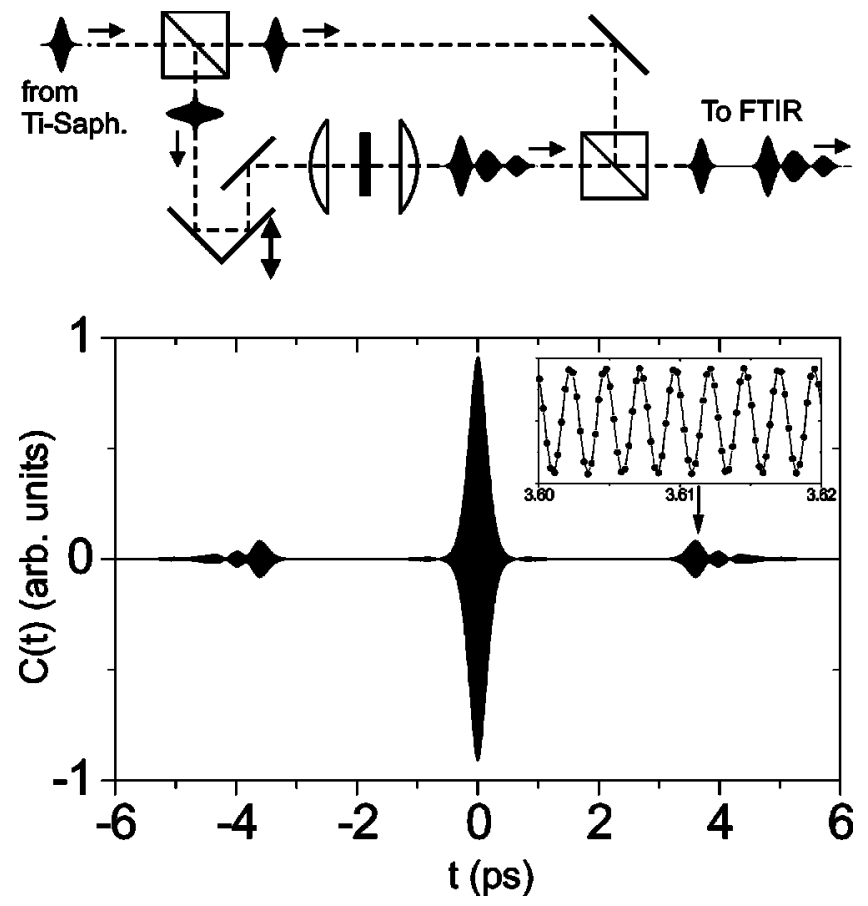

FIG. 1. Cartoon of the experimental setup and an example of a measured autocorrelation function for macroporous $\mathrm{GaP}$ described by Eq. (1). The sample sits at the focal point of two lenses. The interferogram has one large central peak with a symmetric multipeak region on either side. The central peak, left peaks, and right peaks correspond to the first, second, and third terms in Eq. (1), respectively. By deconvoluting the right peaks with the input pulse, the frequency-resolved amplitude and phase can be determined. The inset to the bottom figure shows that single fringes can be resolved with a time resolution of $\sim 0.25 \mathrm{fs}$.

an ultrashort-pulse interferometry technique $[28,29]$. We used polarized bandwidth-limited ultrashort pulses $(\sim 100$ fs) from a Ti:sapphire laser (Tsunami, Spectra Physics) at $739 \mathrm{~nm}$, with a repetition rate of $82 \mathrm{MHz}$. The spectral bandwidth of the pulses was about $1 \%$ of the central frequency. A double-pulsed signal was obtained with a fixed MachZehnder interferometer in which the reference arm is empty and the sample arm contains the scattering sample (Fig. 1). The time lag $t_{0}$ between the pulse and the signal from the sample could be varied using a translatable retroreflector. The beam waist at the sample surface was $\sim 30 \mu \mathrm{m}$. In transmission, diffuse light was collected in the forward direction while in reflection, diffuse light was collected at several degrees from the specular reflection angle allowing the near-normal incident coherent pulse to be blocked. The output from the Mach-Zehnder interferometer passed through an aperture to isolate one speckle spot in the beam output. The resulting beam contained a pair of pulses: an undisturbed pulse followed by a pulse scattered by the sample. The combined beam was sent through a polarizer into a Fourier transform infrared (FTIR) spectrometer (Biorad FTS-60A). The FTIR uses a scanning Michelson interferometer to obtain the field autocorrelation function of the pulse pair by scanning the time delay $t$ between two copies of the pair. 
The complex frequency dependent transfer function $H(f)$ determines how both the magnitude and the phase of each frequency component of the input pulse are changed by the sample to produce the output pulse. I.e., if the frequency spectrum of the pulse is $A(f)$, then the output from a single speckle spot on the sample is $H(f) A(f)$. The FTIR measures the autocorrelate of the reference and the signal from the sample given by

$$
\begin{aligned}
C(t)= & \int\left[|A(f)|^{2}+|A(f) H(f)|^{2}\right] e^{i 2 \pi f} d f \\
& +\int|A(f)|^{2} H(f) e^{i 2 \pi f\left(t+t_{0}\right)} d f \\
& +\int|A(f)|^{2} H(f) e^{i 2 \pi f\left(t-t_{0}\right)} d f .
\end{aligned}
$$

This expression is described in more detail in Ref. [28].

A typical output signal for a single speckle spot from the FTIR is given in Fig. 1. Note that the FTIR resolution is sufficient to resolve single-wave oscillations as shown in the inset, corresponding to a resolution in time of $0.25 \mathrm{fs}$. The source pulse $|A(t)|^{2}=\int|A(f)|^{2} e^{i 2 \pi f t} d f$ was obtained by blocking the sample pulse [Fig. 2(a)]. $|A(f)|^{2}$ was calculated via Fourier transformation. The cross correlated portion of $C(t)$ given by the last two terms of Eq. (1) gives the convolution of the square of the input intensity with the amplitude for light to traverse a single-channel offset by $t_{0}$. The measured values for the last term of $C(t)$ are given in Figs. 2(b)-2(h). In reflection [Figs. 2(b)-2(d)], there is a large initial pulse, that is similar to the source, because most of the diffuse light leaves the sample after traveling just a few mean free paths. By contrast, in transmission [Figs. 2(e) $-2(\mathrm{~g})]$ the signal may show a long rise time. In both cases, the light amplitude passes through zero several times. Such a zero crossing is depicted in Fig. 2(h). Each new speckle spot was obtained by translating the sample $\sim 30 \mu \mathrm{m}$. $C(t)$ was measured for 15-120 speckle spots for each sample.

The average output intensity from the sample, $I_{\text {conv }}(t)$, can be calculated by averaging the squares of the cross correlation functions presented in Fig. 2 over multiple speckle spots,

$$
I_{\text {conv }}(t)=\left\langle\left(\int|A(f)|^{2} H(f) e^{i 2 \pi f\left(t-t_{0}\right)}\right)^{2}\right\rangle .
$$

The variable delay $t_{0}$ could be determined in transmission by removing the sample, and in reflection by unblocking the specularly reflected pulse and measuring its arrival time. An example of a measured $I_{\text {conv }}(t)$ vs $t-t_{0}$ curve in transmission, given in Fig. 3, shows the exponential decay predicted for diffuse propagation. By combining the known input pulse with predictions from diffusion theory, the resulting fit yields the diffusion constant.

To calculate the full complex spectrum $H(f)$, the portion of $C(t)$ containing the cross correlate, $\int|A(f)|^{2} H(f) e^{i 2 \pi f\left(t-t_{0}\right)} d f$, must be separated from the rest of $C(t)$. The data array representing $C(t)$ is set to zero up to the point just before the cross correlate appears and then

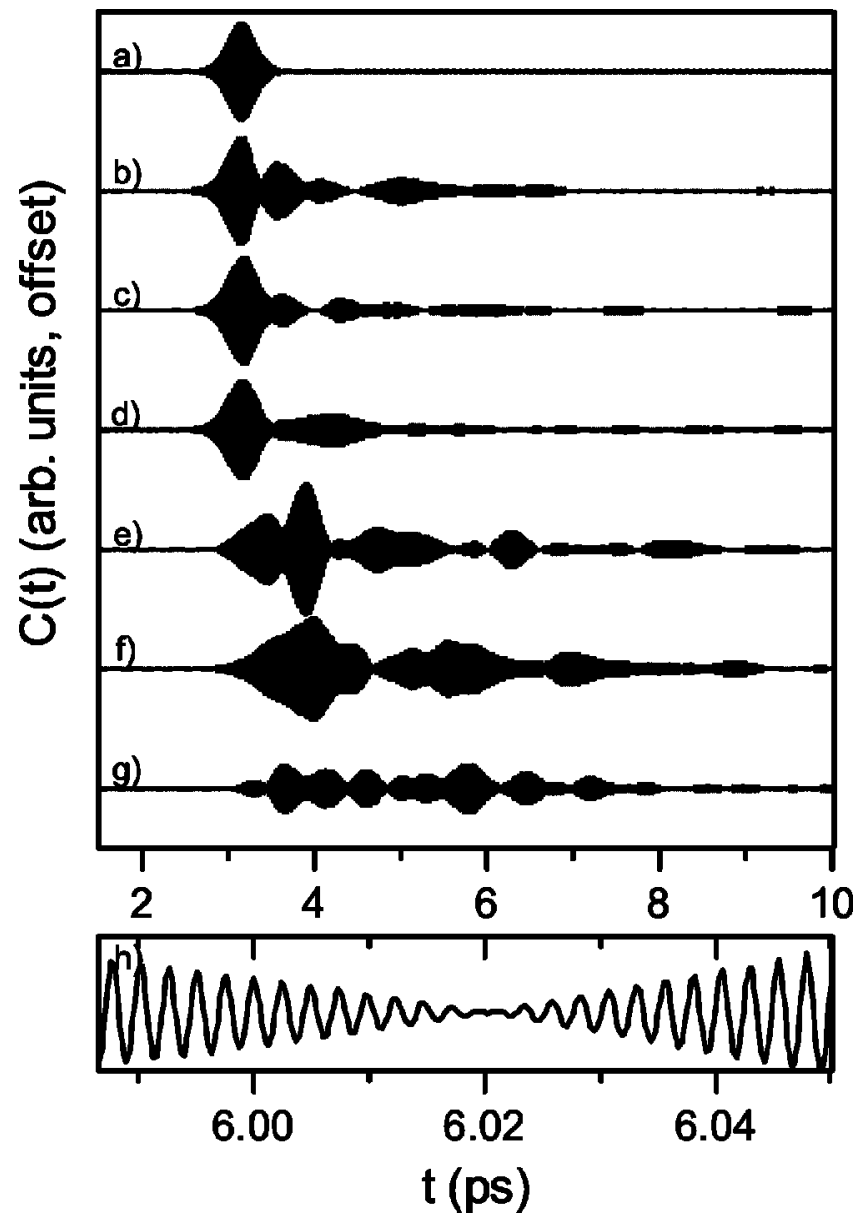

FIG. 2. Examples of the FTIR interferograms for a macroporous GaP sample with different measurement configurations. (a) The source pulse $|A(t)|^{2}$ alone, measured by blocking the beam in the sample leg. (b) $-(\mathrm{g})$ The cross correlation of the source pulse and the diffuse pulse exiting the sample in reflection (b)-(d) and transmission $(\mathrm{e})-(\mathrm{g})$. The bottom plot $(\mathrm{h})$ is a magnification of speckle (e) showing that the intensity passes through zero a number of times.

Fourier transformed. To obtain $H(f)$, the reference $|A(f)|^{2}$ and the time lag $e^{i 2 \pi f t_{0}}$ must be divided out. The resulting complex function $H(f)$ contains both amplitude and phase information as shown in Fig. 4.

In this paper, we will measure the phase statistics for

$$
\phi^{\prime}(f)=(1 / 2 \pi) \partial \phi / \partial f
$$

and

$$
W(f)=|H(f)|^{2} \phi^{\prime}(f)
$$

for diffuse samples. The motivation for these measurements is the recent paper from van Tiggelen et al. [18]. They argue that $\phi^{\prime}$ can be thought of as a single-channel time delay for a given speckle spot. This makes some intuitive sense, since the average of $\phi^{\prime}$ over multiple speckle spots gives the average transport time for light through the sample. The interpretation loses some of its intuitive content when one considers that $\phi^{\prime}$ can be negative for certain frequencies (Fig. 


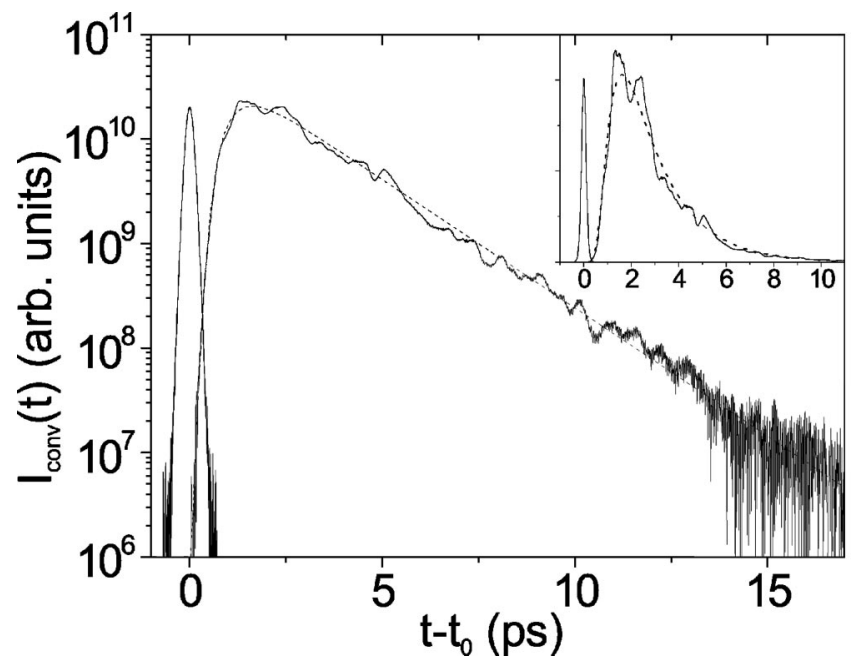

FIG. 3. The diffusely transmitted pulse for a $20-\mu \mathrm{m}$ thick macroporous GaP sample obtained by summing the squares of the FTIR interferograms of 70 different speckles. The narrow pulse centered around the time origin is the incident pulse. The dashed line is Eq. (7), derived from the diffusion equation, with $D$ $=23 \mathrm{~m}^{2} / \mathrm{s}$ and $t_{a}=\infty$. Inset: same data on a linear scale.

4). In the same paper, the statistics for $W(f)$, referred to as the weighted time delay, are also calculated. This quantity is considered fundamental because its sum over all speckle spots in both reflection and transmission gives the density of states for a given frequency. As seen from Fig. 4, both $\phi^{\prime}$ and $W$ can be directly calculated from our measurements.

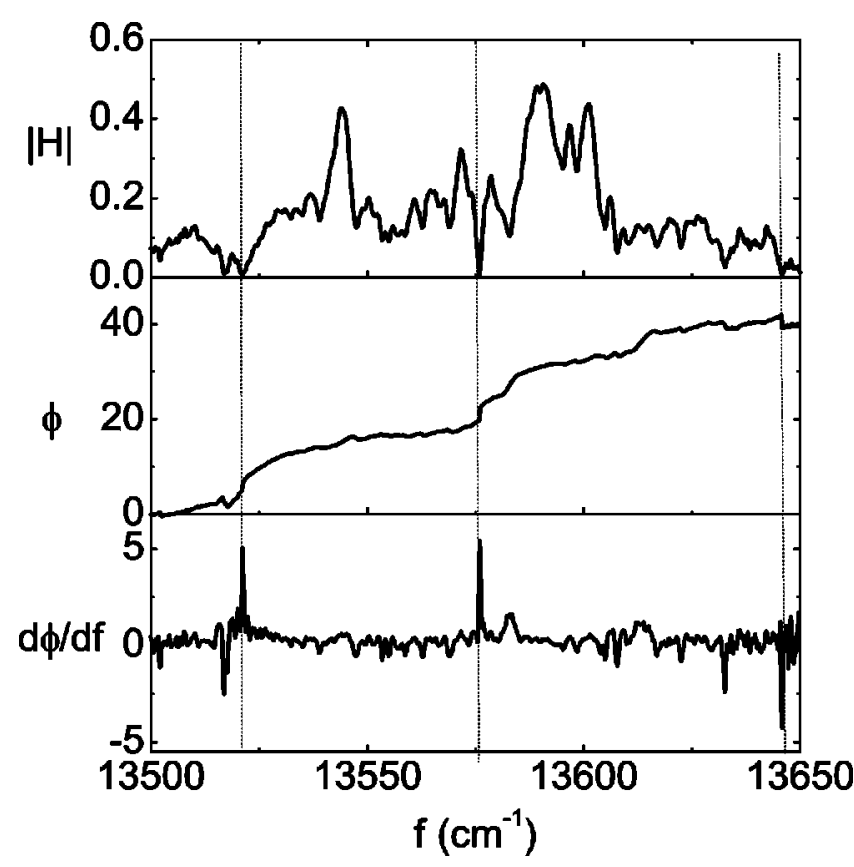

FIG. 4. The frequency dependence of the amplitude $|H|$, phase $\phi=\arg (H)$, and phase derivative $\partial \phi / \partial f$ of the transfer function $H$ for one speckle spot from the $35-\mu \mathrm{m}$ thick macroporous $\mathrm{GaP}$ sample in reflection. The dashed lines mark frequencies for which no light is emitted $(|H|=0)$, causing the phase to show a discontinuity, and the phase derivative to show a strong peak.

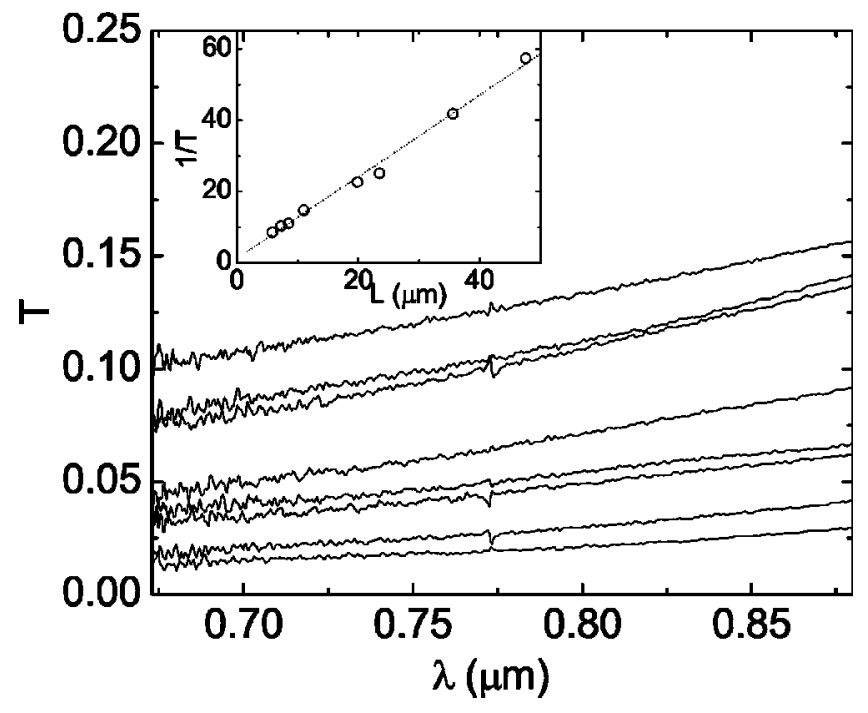

FIG. 5. The total transmission of light $T$ for all of the macroporous $\mathrm{GaP}$ samples as a function of wavelength $\lambda$ and sample thickness $L$. The transmission decreases monotonically with decreasing $\lambda$, indicating that the samples scatter most strongly at the lowest wavelengths measured. The inset gives the value of $1 / T$ for different thicknesses at $\lambda=739 \mathrm{~nm}$, with a fit to Eq. (5) from the diffusion theory.

The derivative $\phi^{\prime}$ was calculated from the slope of a local linear fit to several consecutive values of $\phi$. The value of $\phi^{\prime}$ was checked as a function of the number of points in the linear fit, to check for unwanted smoothing effects. Such effects began to become evident only for more than eight points in the fit. We will compare the statistics of $\phi^{\prime}$ and $W$ with the predictions of Ref. [18]. Such a comparison has not yet been shown for optical waves.

Measurements of the static total transmission $T$ were done with the sample mounted on the entrance opening of a goldcoated (infragold) integrating sphere. The porous side of the sample was illuminated with a collimated halogen lamp. The sphere collected the light transmitted in all directions. An FTIR spectrometer was used to resolve the spectrum.

\section{RESULTS}

\section{A. Static measurements}

The static transport properties were determined from the total transmission $T$ as a function of $L$ and $\lambda$ (Fig. 5). There are no strongly resonant frequencies in the frequency range probed and the total transmission decreases monotonically with decreasing wavelength. Thus the sample scatters light more efficiently at shorter wavelengths, i.e., as the wavelength approaches the pore size. The inverse of the total transmission vs thickness is plotted in Fig. 5 (inset) at $\lambda$ $=739 \mathrm{~nm}$. The linear behavior indicates that the data are described by the classical diffusion theory with negligible absorption [30]:

$$
\frac{1}{T}=L \frac{1}{l\left(1+z_{1}\right)}+\frac{z_{1}+z_{2}}{1+z_{1}} .
$$


Here $z_{1}$ and $z_{2}$ are the extrapolation length ratios on either side of the sample. In our measurements, $z_{1}$ always corresponds to the air-porous GaP interface ("sample side") and $z_{2}$ to the porous $\mathrm{GaP}$-solid $\mathrm{GaP}$ interface ("substrate side").

A detailed study of the surface properties of porous GaP was recently carried out by Gómez Rivas et al. [15]. Following the technique of Vera and Durian [31], they measured the normalized intensity $P(\theta)$ of light exiting the samples at the angle $\theta$. The measured $\theta$ and polarization dependence of $P(\theta)$ was consistent with the model from Ref. [31] in which the diffuse light follows Fresnel's law for internal reflection at the interface as a function of $\theta$ at the interface. This allowed for the measurement of $n_{e}$ for these materials.

Following Ref. [31], $z_{1}$ was calculated for each sample from the expression

$$
z_{1}=\frac{2+\int_{0}^{1} 4 \mu R(\mu) d \mu}{3-\int_{0}^{1} 9 \mu^{2} R(\mu) d \mu},
$$

where $\mu=\cos (\theta)$ and $R(\mu)$ is given by Fresnel's law in reflection at the internal sides of the sample-air interface. As expected, the thinnest sample showed a higher value of $z_{1}$ $\left(z_{1}=3.5 \pm 0.6\right.$ at $\left.L=7.3 \mu \mathrm{m}\right)$ due to the presence of the residual thin solid GaP layer, while the thicker samples gave consistent values of $z_{1}=2.1 \pm 0.3$ and $n_{e}=1.45 \pm 0.15$.

The transport mean free path $l$ at $739 \mathrm{~nm}$ was obtained from the slope of the linear fit in the inset of Fig. 5, yielding $l=0.28 \pm 0.05 \mu \mathrm{m}$. From the same spectrum, $l$ was also obtained at $685 \mathrm{~nm}$ and $822 \mathrm{~nm}$ yielding values of 0.21 $\pm 0.04 \mu \mathrm{m}$ and $0.38 \pm 0.06 \mu \mathrm{m}$, respectively. Thus $l$ increases with wavelength. The value measured at $685 \mathrm{~nm}$ is consistent with that measured by Schuurmans et al. on the same samples [25,27].

The extrapolation length $z_{2}$ does not influence the measurement of the mean free path, but is required to describe the time-resolved measurements presented below. In a static experiment its value is determined, not just by the reflectivity at the interface between porous $\mathrm{GaP}$ and solid $\mathrm{GaP}$, but also by that of the wafer-air interface, which also reflects light back into the sample. The treatment of such a dual interface boundary condition (due to a substrate or a sample cell) was given in Ref. [31] for the static case. In a dynamic experiment, however, the reflection from the second interface is separated in time by twice the interval needed for light to travel the distance between the interfaces; in our case about 8 ps. It, therefore, does not significantly contribute to the primary transmitted pulse. Including only the reflectivity from the first surface and the effective index $n_{e}=1.45$ gives the appropriate value $z_{2}=0.95 \pm 0.1$. For the static experiment, $z_{2}=3.8 \pm 0.3$.

\section{B. Dynamic intensity measurements}

The average intensity as a function of time was measured for the samples first in transmission. The resulting intensity distributions, an example of which is given in Fig. 3, were compared with predictions for transmitted light through a diffusing material [32]:

$$
\begin{aligned}
T(t)= & \frac{-2 \pi D e^{-t / \tau_{a}}}{L_{e}^{2}} \sum_{n=1}^{\infty} n \sin \left(\pi n \frac{l\left(1+z_{1}\right)}{L_{e}}\right) \\
& \times \cos \left(\pi n \frac{L-l\left(1+z_{1}\right)}{L_{e}}\right) \exp \left(-\frac{\pi^{2} n^{2} D t}{L_{e}^{2}}\right) .
\end{aligned}
$$

Here, $D$ is the diffusion coefficient, $\tau_{a}$ is the time required for light to be absorbed in the samples, and $L_{e}=L+l\left(z_{1}\right.$ $\left.+z_{2}\right)$. Recent measurements of much thicker ( $L$ $=350 \mu \mathrm{m}$ ), somewhat less strongly scattering, porous $\mathrm{GaP}$ samples gave $\tau_{a}=590 \mathrm{ps}$, which is two orders of magnitude larger than the relevant time scales for the experiments here [7]. Increasing the scattering strength should not substantially change $\tau_{a}$. Also, the total transmission measurements showed no evidence of absorption. Therefore, it is safe to assume that $\tau_{a}$ is effectively infinity for the time scales considered here. To obtain a fit, Eq. (7) was convoluted with the input pulse shape. A sample fit is given in Fig. 3 using $D$ $=23 \mathrm{~m}^{2} / \mathrm{s}$. Good fits were obtained for samples of any thickness.

At long times, Eq. (7) predicts a single exponential decay with a rate $1 / \tau_{d}$ given by

$$
\frac{1}{\tau_{d}}=\frac{1}{\tau_{a}}+\frac{\pi^{2} D}{L_{e}^{2}}
$$

which allows for a measurement of the diffusion constant $D$ from the slope of the long tail in the log-linear plot. Repeated measurements showed that diffusion coefficients measured from the decay of the tail of the transmitted pulse were reproducible to within $6 \%$.

In reflection, the expression for intensity as a function of time given by diffusion theory is

$$
\begin{aligned}
R(t)= & \frac{3 D}{l L_{e}} \exp \left(-\frac{t}{\tau_{a}}\right) \sum_{n=1}^{\infty}\left\{\exp \left(-\frac{D \pi^{2} n^{2}}{L_{e}^{2}} t\right)\right. \\
& \left.\times\left[1-\cos \left(\frac{l+z_{1}}{L_{e}} 2 \pi n\right)\right]\right\} .
\end{aligned}
$$

For short times $\left[t<L_{e}^{2} /\left(\pi^{2} D\right)\right]$, Eq. (9) reduces to a simple power law decay with $R(t) \propto t^{-3 / 2}$. At long times, Eq. (9) predicts the same single exponential decay as in transmission with the exponent given by Eq. (8).

The measured $I_{\text {conv }}(t)$ in reflection for an $L=35 \mu \mathrm{m}$ thick sample is given in Fig. 6. This plot shows the two regions of decay. At long times, the decay is exponential, as seen by the linear behavior on the log-linear plot. In the inset, the same data are given on a log-log plot. In the interval between $\sim 0.5$ and $10 \mathrm{ps}$, power law behavior is observed. The power law behavior breaks down at short times, 


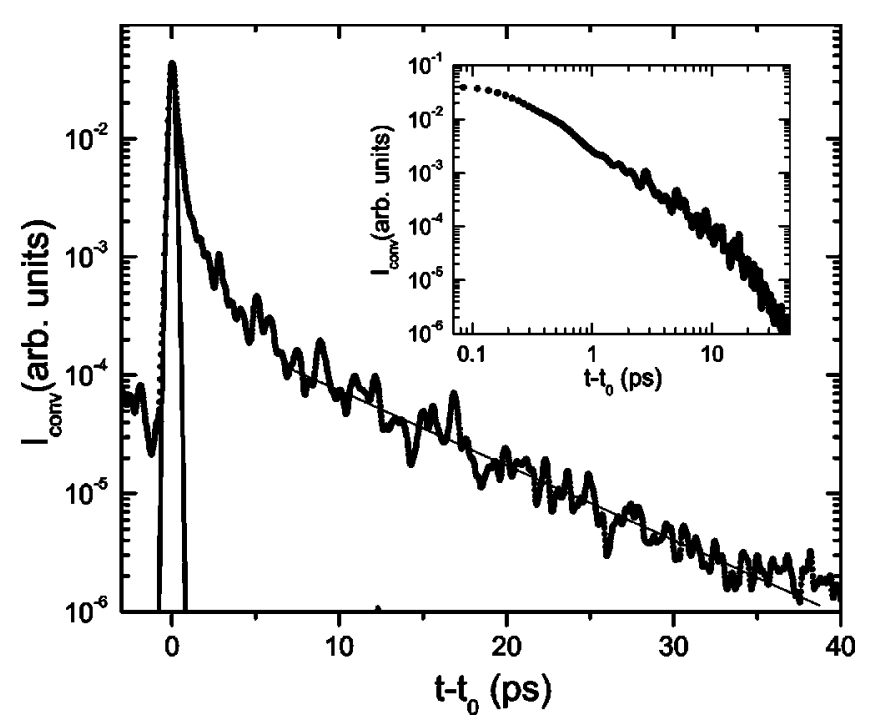

FIG. 6. Intensity of reflected light as a function of time for the 35- $\mu$ m-thick macroporous GaP sample. The log-linear plot makes clear the long time exponential decay. The narrow pulse at $t-t_{0}$ $=0$ is the source pulse. The inset is the same data on a log-log plot, showing the power law behavior at short times. The rounding at $t$ $-t_{0}<0.3$ ps occurs because the intensity is convoluted with the input pulse.

because the measured pulse is a convolution of the input pulse with Eq. (9), and at long times where the exponential behavior sets in.

The exponent of the power law decay in reflection is of particular importance in searching for clues of localization. A recent paper by Titov and Beenakker describes a transition of the power law exponent from $-3 / 2$ to -2 with the onset of localization [33]. To resolve the intensity at the shortest possible times and thus minimize the error on the power law fit, the signal in transmission was deconvoluted with the incident pulse. This was achieved by first calculating $H(f)$ as described above, and then inverse Fourier transforming and squaring the result. The result $[I(t)]$ extends the power law behavior to $\sim 0.1 \mathrm{ps}$ as seen in Fig. 7 . This data can be fit to a linear function of the log-log plot giving a slope of -1.55 \pm 0.1 . This is consistent with the diffusion theory and thus shows no evidence of localization within the uncertainty of our experiment. Other thick samples gave the same result for the power law exponent, while thinner samples did not offer enough of a time window to allow for meaningful fits. All of the samples showed exponential behavior at long times as in the transmission measurements.

In both transmission and reflection, the decay time $\tau_{d}$ depends on the diffusion constant and the absorption length. Plotting $1 / \tau_{d}$ against $1 / L_{e}^{2}$ gives an indication of the dependence of $D$ on thickness and the value of $L_{a}$. A linear function is predicted by the diffusion theory while a function of increasing slope is predicted in the presence of localization. The inverse of $\tau_{a}$ corresponds to the intercept of the linear function with the $y$ axis. The resulting plot (Fig. 8, circles) shows a linear relationship between $1 / \tau_{d}$ and $1 / L_{e}^{2}$ as predicted by the diffusion theory. The fit gives $D=22$

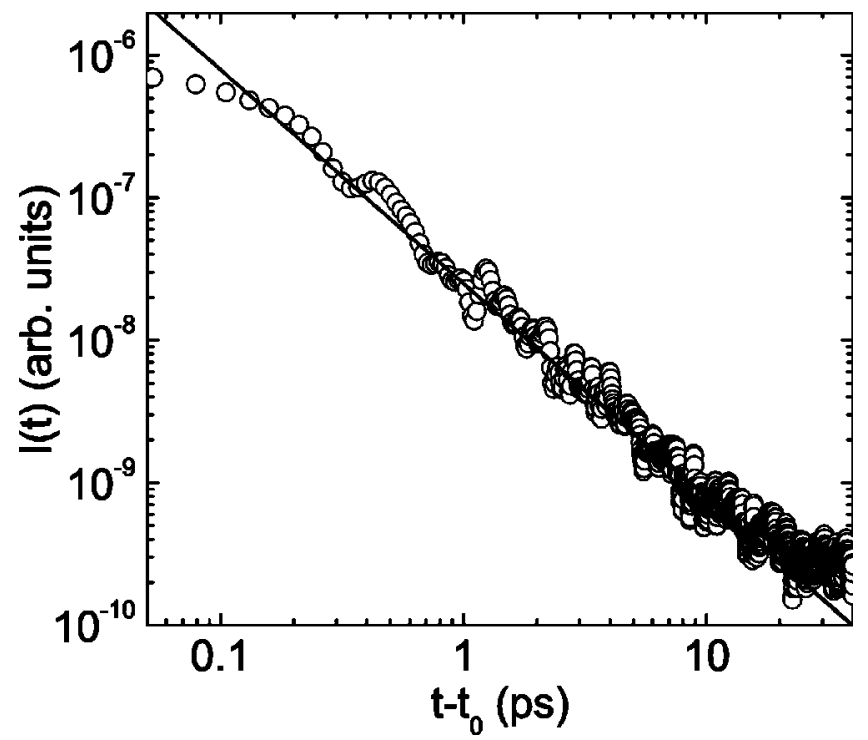

FIG. 7. Intensity of reflected light as a function of time in reflection for the $35-\mu \mathrm{m}$ macroporous $\mathrm{GaP}$ sample, with the input pulse deconvoluted from the signal. The solid line demonstrates the power law behavior, $I(t) \propto\left(t-t_{0}\right)^{-1.55 \pm 0.1}$.

$\pm 4 \mathrm{~m}^{2} / \mathrm{s}$. The intercept is zero to within experimental uncertainty, consistent with an unmeasurably large $\tau_{a}$. The large uncertainty for the thinnest samples (where $1 / L_{e}^{2}$ is large) occurs because of uncertainty in the measurement of the sample thickness and in the determination of $z_{1}$ and $z_{2}$. For thin samples, the extrapolation lengths make a nonnegligible contribution to $L_{e}$. This fact highlights the importance of careful characterization of the sample surface, especially in thin, strongly scattering samples.

The energy velocity can be obtained from the relation $v_{e}=3 \mathrm{D} / l$. The resulting value $v_{e}=(0.79 \pm 0.15) c$ is much higher than that seen in other strongly scattering samples $[16,35]$. This result is addressed in Sec. IV.

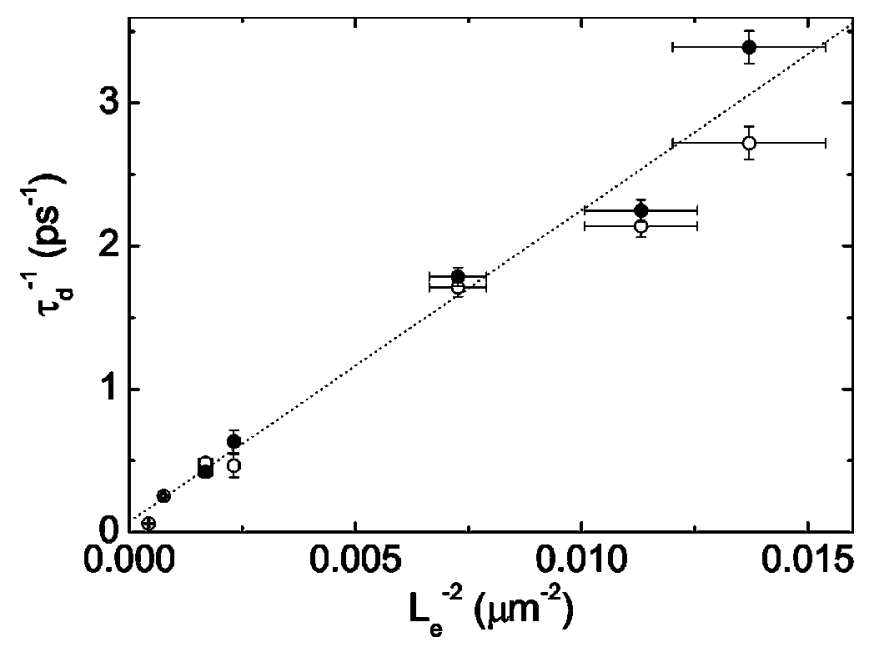

FIG. 8. $\tau^{-1}$ vs $L_{e}^{-2}$ in transmission (filled circles) and reflection (open circles) as measured from the long time exponential tails of $I(t)$ for the macroporous $\mathrm{GaP}$ samples. The slope of the linear fit yields $D=22 \pm 4 \mathrm{~m}^{2} / \mathrm{s}$. 


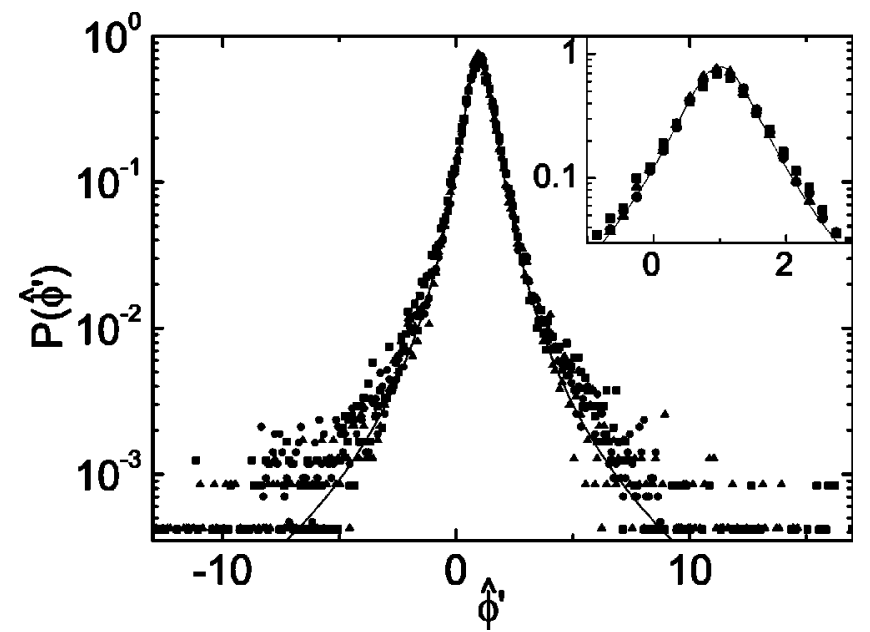

FIG. 9. The probability distribution for the phase derivative $P\left(\hat{\phi}^{\prime}\right)$ in transmission for macroporous GaP samples with thicknesses $L$ of 42 (circles), 20 (squares), and 5 (triangles) $\mu \mathrm{m}$. The 5 - $\mu \mathrm{m}$ sample had not been photoanodically etched, thus it had a higher porosity and mean free path $l$. The results show that this distribution is independent of $L$ or $l$. The solid line is Eq. (10) with $Q=0.4$. The inset shows the quality of the fit at the peak.

\section{Dynamic phase measurements}

Two statistical properties were measured in transmission, the normalized probability distributions for $\hat{\phi}^{\prime}=\phi^{\prime} /\left\langle\phi^{\prime}\right\rangle$, and $\hat{W}=W /\langle W\rangle$. The bracketed quantities are averages over 15-120 speckle spots and over the narrow frequency range of the pulse. These distributions are predicted to have the following functional form [18]:

$$
\begin{gathered}
P\left(\hat{\phi}^{\prime}\right)=\frac{1}{2} \frac{Q}{\left[Q+\left(\hat{\phi}^{\prime}-1\right)^{2}\right]^{3 / 2}}, \\
P(\hat{W})=\frac{1}{\sqrt{Q+1}} \exp \left(\frac{-2|\hat{W}|}{\operatorname{sgn}(\hat{W})+\sqrt{Q+1}}\right) .
\end{gathered}
$$

The parameter $Q$ depends on the material properties of the sample. In transmission, $Q_{T}=2 / 5$ in the absence of absorption, and decreases monotonically with increasing absorption. $Q_{T}$ is expected to be independent of $L, D$, and $l$. In reflection in the absence of absorption [34],

$$
Q_{R}=L_{e} \frac{2}{5} \frac{1}{l\left(1+z_{1}\right)} .
$$

Thus $Q_{R}$ is predicted to increase linearly with thickness. Such a measurement should allow for the measurement of the mean free path. $Q_{R}$ is also sensitive to surface properties in reflection via $z_{1}$.

Results for $P\left(\hat{\phi}^{\prime}\right)$ in transmission for several samples of different thickness are shown in Fig. 9. Also included is one sample that had not been photoanodically etched, and therefore has a smaller pore size and roughly twice the mean free path. The distribution for all of these samples collapses onto one curve. Also plotted is Eq. (10) with $Q_{T}=0.4$. Our data

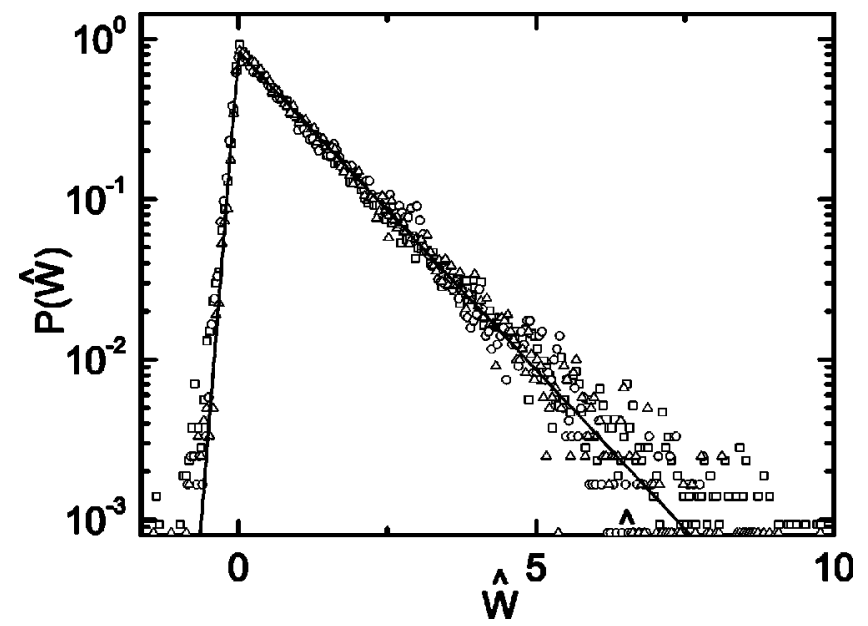

FIG. 10. $P(\hat{W})$ for the same three samples as in Fig. 9. The solid line is Eq. (11) with $Q=0.4$.

show excellent agreement with the predictions over three decades, confirming the theories of van Tiggelen et al., and showing that absorption is minimal in these samples. The resulting distribution was found to be invariant with respect to the histogram bin size. Similar excellent agreement between data and theory was obtained for $P(\hat{W})$ assuming no absorption as seen in Fig. 10. These results also demonstrate that the form of the distributions are independent of $L$ or $l$, as predicted.

Results for $P\left(\hat{\phi}^{\prime}\right)$ in reflection for two different thicknesses are shown in Fig. 11. The predicted distribution given in Eq. (12) again fits the data well, with agreement over 2.5 decades, allowing $Q_{R}$ to be measured for different samples of different thicknesses. The distribution for the thicker sample $(L=47 \mu \mathrm{m})$ is much wider than that for the thinner ( $L=8.5 \mu \mathrm{m})$ as expected. The resulting fits to Eq. (10) give values of $Q_{R}=43$ and $Q_{R}=4.3$ for the thick and thin samples, respectively. Using the measured values of $l$ and $z_{1}$,

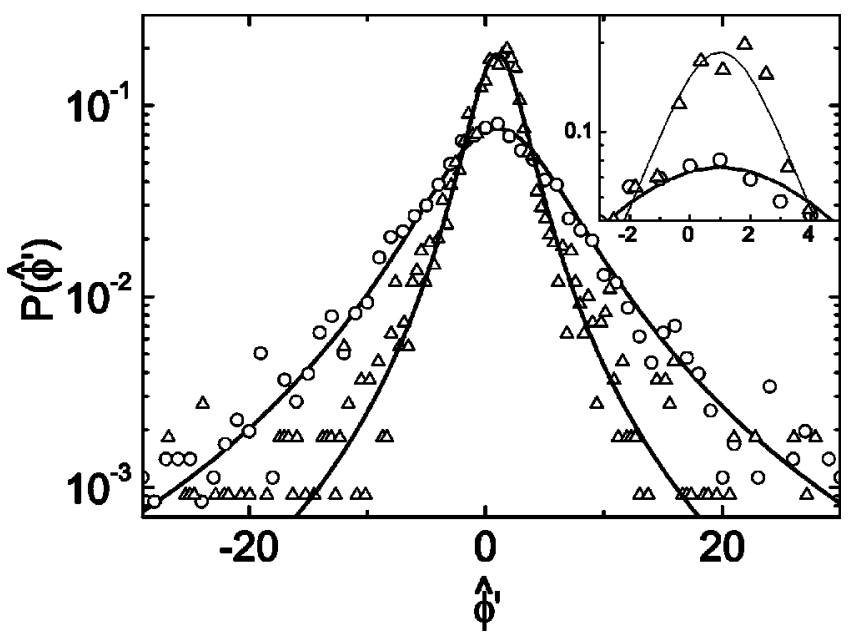

FIG. 11. $P\left(\hat{\phi}^{\prime}\right)$ in reflection for macroporous GaP samples with thicknesses $L=47 \mu \mathrm{m}$ (circles) and $L=8.5 \mu \mathrm{m}$ (triangles). The solid lines are fits to Eq. (10) with the results $Q_{R}=43$ and $Q_{R}$ $=4.3$. The distribution width is strongly thickness dependent. 
TABLE I. $l, D$, and $v_{e}$ for different sample geometries.

\begin{tabular}{lccccccc}
\hline \hline Material & Geometry & $n$ & $\lambda(\mu \mathrm{m})$ & $l(\mu \mathrm{m})$ & $D\left(\mathrm{~m}^{2} / \mathrm{s}\right)$ & $v_{e} / c$ & Ref. \\
\hline Titania & Powder & 2.6 & 0.633 & $0.57 \pm 0.05$ & $11.7 \pm 1$ & $0.16 \pm 0.03$ & {$[16]$} \\
Germanium & Powder & 4.0 & 8 & $3.8 \pm 0.2$ & 26 & 0.18 & {$[35]$} \\
$\mathrm{GaP}$ & Macroporous sponge & 3.3 & 0.74 & $0.28 \pm 0.05$ & $23 \pm 3$ & $0.79 \pm 0.15$ & Current paper \\
\hline \hline
\end{tabular}

Eq. (10) predicts $Q_{R}=21$ and $Q_{R}=3.8$ for the thick and thin samples, respectively. Thus while the thinner sample shows reasonable agreement for the value of $Q_{R}$, the thicker samples deviate from the predicted value. Measurements on the other samples show good agreement for the shape of the distribution, but such a large scatter in the measured $Q_{R}$ that no systematic dependence of $Q_{R}$ on thickness could be determined.

\section{DISCUSSION}

Our results for these samples agree with the diffusion theory in the time domain. In both reflection and transmission, $I(t)$ shows diffusionlike profiles. At short times, power law decay of $I(t)$ over two decades with an exponent of $1.55 \pm 0.1$ is observed in reflection. This also agrees with diffusion theory. The diffusion constant is independent of sample thickness and there is no measurable absorption. The phase statistics also show excellent agreement with the diffusion theory. In transmission, the distributions of $\hat{\phi}^{\prime}$ and $\hat{W}$ agree with the predictions of van Tiggelen et al. in both the functional form and width of the distribution. As predicted, $\hat{\phi}^{\prime}$ and $\hat{W}$ were found to be independent of $L, l$, and $z_{1}$ in transmission. In reflection, the functional form of $\hat{\phi}^{\prime}$ also fits the predictions of van Tiggelen et al. The distributions in reflection were also found to be thickness dependent, as predicted.

It is interesting to compare the results for $D, l$, and $v_{e}$ for macroporous $\mathrm{GaP}$ with results from strongly scattering powders. The dynamic and static scattering properties of both titania and germanium have been previously measured. The results, summarized in Table I along with the results for GaP, show that $v_{e}$ is roughly a factor of 5 times lower in strongly scattering powders than in macroporous GaP. Thus, for example, the titania powders show a lower diffusion constant than $\mathrm{GaP}$ even though the mean free path in titania is more than twice that measured for GaP. All of the samples show a high-index contrast, and a mean free path of the order of the wavelength. The main difference between the samples is the mesoscopic topology, which is a likely explanation for the large difference in $v_{e}$. The low energy velocity in powders has been previously explained [16] as arising from Mie-like resonances. The grains of powder were treated as independent Mie scatterers. As such, the scatterers show strong resonances that cause the scattering time to increase dramatically. However, a Mie sphere of air in a high-index material, which is perhaps equally comparable to macroporous $\mathrm{GaP}$, does not show such strong resonances. In fact, it is difficult to define independent scatters given the bicontinuous porous structure of macroporous $\mathrm{GaP}$. Thus, it is possible that the resonant effect on the scattering time is absent. It is interesting also to note that the effective phase velocity, given by $c / n_{e}$ $\approx c / 1.45$, is also higher than expected from mean field approximations of $n_{e}$ [15]. While this gives a qualitative explanation for the unique transport properties of these samples, a quantitative explanation for the high value of $v_{e}$ measured here is needed.

The agreement with diffusion theory measured in the time domain is consistent with the results for total transmission seen by Schuurmans et al. [25]. However, the subtle effects for long light paths seen in the previously measured backscatter cones [27] are not manifested in the time-resolved measurements presented here. This does not rule out the possibility that the localization effects reported by Schuurmans et al. exist at a level below our experimental uncertainty, since the enhanced backscatter cone rounding is apparently quite sensitive to anomalies in the path length distribution [36]. Thus we can neither verify nor disprove the localization explanation given in Ref. [27].

The measured $Q_{R}$ showed some deviation from Eq. (12). While there was agreement for some samples, other samples showed as much as a $50 \%$ difference between the measured and predicted $Q_{R}$. In reflection, much of the light exits the sample within the first few scattering events. With $l$ $=0.28 \mu \mathrm{m}$, much of the light is probing the porous region within less than $1 \mu \mathrm{m}$ from the surface. While we made a considerable effort to characterize and optimize the surface of these samples, the phase statistics could be a particularly sensitive test of the surface properties and sample dependent surface anomalies. A second difference between measuring in reflection and in transmission is that $t_{0}$ is more difficult to measure in reflection. As mentioned above, much of the light escapes the sample just after $t_{0}$. Thus the importance of using the correct $t_{0}$ is amplified in reflection. It is possible that diffusion theory breaks down in reflection due to the close proximity of the surface to much of the light intensity. Resolving the problem of these anomalous reflection statistics could lead to a better understanding of light propagation and surface properties of strongly scattering samples.

Finally, we would like to highlight the importance of careful measurement of surface properties and the effective index of refraction when characterizing strongly scattering materials. A strong index contrast between the bulk material and the outside environment means that the extrapolation length can be several times the mean free path due to internal reflection of diffuse light. This can lead to anomalously long decay times in time-resolved measurements, where light is trapped inside the material due to the high internal reflection. As seen in Ref. [15], the effective index of refraction of these 
materials, while measurable, cannot be calculated a priori using presently available effective medium theories. This is understandable, since $\lambda$ is of the order of the internal length scale of these samples.

To conclude, we have demonstrated that time-resolved, phase-sensitive measurements can be carried out on strongly scattering gallium phosphide samples. We find an unusually high $v_{e}$ compared with powders, which may be due to the bicontinuous spongelike geometry of the samples. We find no evidence of localization. Most of our results agree with the diffusion theory, though some questions with respect to the phase statistics in reflection remain to be resolved. We are currently improving our processing techniques for $\mathrm{GaP}$ and hope to improve both the scattering strength and the uniformity of the surface. We also plan to further examine the phase statistics to, for example, measure correlation in the phase-sensitive quantities.

\section{ACKNOWLEDGMENTS}

We gratefully acknowledge T. Hijmans for his help in understanding these and other experiments on these samples, B. van Tiggelen for providing the calculations for phase statistics in reflection and for many other useful discussions, D. $\mathrm{H}$. Dau for his measurements of the angle-resolved transmission, and F. J. P. Schuurmans, D. Vanmaekelbergh, and J. van de Lagemaat for the preparation of the samples. This work was part of the research program of the "Stichting voor Fundamenteel Onderzoek der Materie (FOM)" which was financially supported by the "Nederlandse Organisatie voor Wetenschappelijk Onderzoek (NWO)."
[1] A. Yodh and B. Chance, Phys. Today 48(3), 34 (1995).

[2] G. Maret and P.E. Wolf, Z. Phys. B: Condens. Matter 65, 409 (1987); D.J. Pine, D.A. Weitz, P.M. Chaikin, and E. Herbolzheimer, Phys. Rev. Lett. 60, 1134 (1988).

[3] A.F. Koenderink and W.L. Vos (unpublished); A.F. Koenderink, M. Megens, G. van Soest, W.L. Vos, and A. Lagendijk, Phys. Lett. A 268, 104 (2000).

[4] S. John, Phys. Rev. Lett. 58, 2486 (1987).

[5] M.H. Kao, K.A. Jester, A.G. Yodh, and P.J. Collings, Phys. Rev. Lett. 77, 2233 (1996).

[6] D.S. Wiersma, A. Muzzi, M. Colocci, and R. Righini, Phys. Rev. Lett. 83, 4321 (1999).

[7] P.M. Johnson, B.P.J. Bret, J. Gómez Rivas, J.J. Kelly, and A. Lagendijk, Phys. Rev. Lett. 89, 243901 (2002).

[8] P. Sheng, Introduction to Wave Scattering, Localization, and Mesoscopic Phenomena (Academic Press, New York, 1995).

[9] I. Freund, M. Rosenbluh, and S. Feng, Phys. Rev. Lett. 61, 2328 (1988); N. Garcia and A.Z. Genack, ibid. 63, 1678 (1989); M.P. van Albada, J.F. de Boer, and A. Lagendijk, ibid. 64, 2787 (1990).

[10] F. Scheffold and G. Maret, Phys. Rev. Lett. 81, 5800 (1998).

[11] P.W. Anderson, Phys. Rev. 109, 1492 (1958); Philos. Mag. B 52, 505 (1985).

[12] N. Garcia and A.Z. Genack, Phys. Rev. Lett. 66, 1850 (1991); A.Z. Genack and N. Garcia, ibid. 66, 2064 (1991).

[13] D.S. Wiersma, P. Bartolini, A. Lagendijk, and R. Righini, Nature (London) 390, 671 (1997).

[14] F. Scheffold, R. Lenke, R. Tweer, and G. Maret, Nature (London) 398, 206 (1999).

[15] J. Gómez Rivas, D.H. Dau, A. Imhof, R. Sprik, B.P.J. Bret, P.M. Johnson, T.W. Hijmans, and A. Lagendijk, Opt. Commun. 220, 17 (2003).

[16] M.P. van Albada, B.A. van Tiggelen, A. Lagendijk, and A. Tip, Phys. Rev. Lett. 66, 3132 (1991).
[17] K. Busch and C.M. Soukoulis, Phys. Rev. Lett. 75, 3442 (1995).

[18] B.A. van Tiggelen, P. Sebbah, M. Stoytchev, and A.Z. Genack, Phys. Rev. E 59, 7166 (1999).

[19] A.Z. Genack, P. Sebbah, M. Stoytchev, and B.A. van Tiggelen, Phys. Rev. Lett. 82, 715 (1999).

[20] V.S. Letokhov, Zh. Eksp. Teor. Fiz. 53, 1442 (1967) [Sov. Phys. JETP 26, 835 (1968)].

[21] Y. Kuga and A. Ishimaru, J. Opt. Soc. Am. A 8, 831 (1984); M.P. van Albada and A. Lagendijk, Phys. Rev. Lett. 55, 2692 (1985); P.E. Wolf and G. Maret, ibid. 55, 2696 (1985).

[22] E. Abrahams, P.W. Anderson, D.C. Licciardello, and T.V. Ramakrishnan, Phys. Rev. Lett. 42, 673 (1979).

[23] A.F. Ioffe and A.R. Regel, Prog. Semicond. 4, 237 (1960).

[24] A.A. Chabanov, M. Stotchev, and A.F. Genack, Nature (London) 404, 850 (2000).

[25] F.J.P. Schuurmans, D. Vanmaekelbergh, J. van de Lagemaat, and A. Lagendijk, Science 284, 141 (1999).

[26] J. Gomez Rivas, A. Lagendijk, R.W. Tjerkstra, D. Vanmaekelbergh, and J.J. Kelly, Appl. Phys. Lett. 80, 4498 (2002).

[27] F.J.P. Schuurmans, M. Megens, D. Vanmaekelbergh, and A. Lagendijk, Phys. Rev. Lett. 83, 2183 (1999).

[28] R.H.J. Kop and R. Sprik, Rev. Sci. Instrum. 66, 5459 (1995).

[29] R.H.J. Kop, P. de Vries, R. Sprik, and A. Lagendijk, Phys. Rev. Lett. 79, 4369 (1997).

[30] D.J. Durian, Phys. Rev. E 50, 857 (1994).

[31] M.U. Vera and D.J. Durian, Phys. Rev. E 53, 3215 (1996).

[32] A.Z. Genack and J.M. Drake, Europhys. Lett. 11, 331 (1990).

[33] M. Titov and C.W.J. Beenakker, Phys. Rev. Lett. 85, 3388 (2001).

[34] B.A. van Tiggelen (private communication).

[35] J. Gómez Rivas, R. Sprik, A. Lagendijk, L.D. Noordam, and C.W. Rella, Phys. Rev. E 63, 046613 (2001).

[36] B.A. van Tiggelen, A. Lagendijk, and D.S. Wiersma, Phys. Rev. Lett. 84, 4333 (2000). 